\title{
Simulating eco-evolutionary processes in an obligate pollination model with a genetic algorithm
}

\author{
Roger Cropp · John Norbury
}

Received: date / Accepted: date

\begin{abstract}
Pollination interactions are common and their maintenance is critical for many food crops upon which human populations depend. Pollination is a mutualism interaction; together with predation and competition, mutualism makes up the triumvirate of fundamental interactions that control population dynamics. Here we examine pollination interactions (nectar reward for gamete transport service) using a simple heuristic model similar to the Lotka-Volterra models that have underpinned our understanding of predation and competition so effectively since the 1920s. We use a genetic algorithm to simulate the eco-evolutionary interactions of the plant and pollinator populations and examine the distributions of the parameter values and zero isoclines to infer the relative ubiquity of the various eco-evolutionary outcomes possible in the model. Our results suggest that trade-offs between costs and benefits for the pollinator may be a key component of obligate pollination systems in achieving adaptive success creating and stably occupying mutualist niches.
\end{abstract}

Keywords obligate mutualism · finite resource $\cdot$ coevolution · genetic algorithm

\section{Introduction}

Pollination is a mutualist interaction between a plant and an animal that is a fundamental component of human agriculture, and is also a widespread interaction in the natural world $[4,5,21,24,26]$. In the first major review of mutualism Boucher [4] claimed that roughly half of all population interactions amongst green (i.e. photosynthesising) species are co-operative, catalytic or in some way mutualist in that each population benefits from the presence of the other. A recent review

R. Cropp

Griffith School of Environment, Griffith University, Nathan, Queensland, 4111, Australia

Tel.: +61-7-37354036

E-mail: r.cropp@griffith.edu.au

J. Norbury

Mathematical Institute, University of Oxford, Andrew Wiles Building, ROQ, Woodstock Road, Oxford OX2 6GG, UK. 
by Bronstein [5] suggested that mutualist interactions are now perceived to be universal, involving virtually every species on Earth. However, our understanding of mutualist interactions has been hampered by a lack of simple models similar to the Lotka-Volterra models $[17,28]$ that have so well explained competition and predation [14]. Mutualist interactions are distinct from most other population interactions in that the benefits acquired often occur through indirect transfers of mass, although interactions such as pollination may also include direct transfers of mass. Mutualist interactions are obligate when one population is dependent upon the other for its survival, or facultative when one population benefits from the presence of the other, but can survive in its absence. The objective of this work is to examine the trade-offs involved in a plant population becoming obligate upon a pollinating population, that might lead to mutualist niches to facilitate the survival of both populations.

Direct population interactions such as predator-prey interactions are constrained by the axiom that a predator cannot gain more mass than its prey loses. Mutualist interactions involve the indirect acquisition of benefits represented by transfers of mass that are facilitated, but not directly provided, by their mutualist benefactor. These indirect transfers of mass reflect the increased fitness of the benefiting population measured by its sequestration of an increased share of the total resources in the system. In the case of pollination interactions, such an increased share of resources may result from supplementary benefits associated with pollination. For example, mutualist benefits might accrue when the pollinating population improves the fitness of the plant population by increasing its geographical spread and competitive ability with other species through higher seed production. The acquisition of resources by a population due to indirect transfers of mass reduces the quantum of resources available to its competitors (and itself) by the same amount, and must be carefully accounted for to ensure that populations do not increase to infinity in "an orgy of mutual benefaction" [19]. The Conservative Normal approach to ecosystem modelling [6] provides a set of simple rules that extends the common consumer-resource modelling approach [18] to ensure that such unrealistic outcomes are not possible. This allows an extension of the great heuristic value of simple Lotka-Volterra models to investigate population interactions such as mutualism.

It is well-known that organisms may modify their biotic and abiotic environment, and alter the selection pressures that operate on themselves and other populations that interact with them [29]. It is a commonly held view that ecosystems evolve to maximise their total biomass, for example Dubois [11] considers that "The general law is that the species evolves so that its biomass increases: evolution tends to maximise the biomass". Similarly, de Mazancourt et al. [10] write "When plant recycling occurs, but both herbivore recycling and migration of mineral nutrient are absent, evolution simply leads toward those strategies that maximize biomass or primary production, depending on the scenario used". Further, maximising total biomass in an ecosystem is approximately synonymous with maximising exergy, a commonly proposed goal function for ecosystem evolution from a thermodynamics perspective (for example: Jorgenson and Mejer [15] suggest that "The thermodynamics of evolution states that an ecosystem evolves towards maximum biomass."). In the spirit of these views, we shall adopt the heuristic that populations in ecosystems adapt to maximise their biomass in our approach. 
Here, we simulate eco-evolutionary processes of two populations in a simple pollination system using a Lotka-Volterra model cast in the Conservative Normal (CN) framework that constitutes the simplest representation of the population interactions. The CN framework is set out in [6] and provides conditions on parameter relationships that we use to define the mutualist interactions. It further ensures that ecosystem models have sensible behaviours and properties, and in the mutualism context prevents models from having solutions where populations can go to infinity. We use an explicit obligation term $[8,12]$ to represent the plant population's dependence on the pollinating population to be able to grow. We utilise a genetic algorithm to simulate adaptation of the plant and pollinator populations and examine the distributions of the parameter values and zero isoclines to infer the relative ubiquity of the various eco-evolutionary outcomes possible in the model. These are indicated by the densities of the distributions of the populations' zero isoclines in the ecospace and indicate heuristics for theoretical probabilities for observing pollination systems with different population attributes.

\section{The Pollination Model}

We consider obligate pollination between a plant population $\left(x_{1}\right)$ that consumes inorganic nutrient $N$ to grow, but that depends on the presence of a pollinator (often an insect) population $\left(x_{2}\right)$ to be able to reproduce. The pollinator, which feeds solely on nectar provided by the plant, provides a transport service, moving pollen inadvertently acquired during feeding from one plant to another. The biomasses of the living populations $x_{1}$ and $x_{2}$, and the inorganic nutrient pool $N$, are measured as the fraction of the total limiting nutrient in the system that they contain. We assume that the ecosystem recycles $100 \%$ of its limiting nutrient (most ecosystems recycle over $90 \%$ of their limiting nutrient [27]) and is closed to influxes and effluxes of this nutrient, but is open to flows of all other nutrients and energy.

We examine a simple generic model of an obligate pollination interaction (see the Supplementary Material for further details), of a plant population $x_{1}$ dependent on a pollinator population $x_{2}$ :

$$
\begin{aligned}
& \dot{x}_{1}=x_{1} f_{1}\left(x_{1}, x_{2}\right)=x_{1}\left(r_{1} \frac{x_{2}}{x_{2}+\epsilon_{2}}-a_{11} x_{1}-a_{12} x_{2}\right) \\
& \dot{x}_{2}=x_{2} f_{2}\left(x_{1}, x_{2}\right)=x_{2}\left(r_{2}-a_{21} x_{1}-a_{22} x_{2}\right) .
\end{aligned}
$$

The substitution $N=1-x_{1}-x_{2}$ has been made for the inorganic nutrient in the plant equation prior to imposing obligation, reflecting the approximation made in the CN framework that the mass of the limiting resource is conserved and recycled within the system. As noted above, this is a reasonable approximation, and creates an ecospace $E \equiv\left\{x_{i} ; 0<x_{i}, x_{1}+x_{2}<1\right\}$ within which all realistic solutions lie. The upper bound of $E$, defined by $x_{1}+x_{2}=1$ provides a natural constraint on the quantum of mutualist benefit that may be accrued by either population, and prevents May's "orgy of mutual benefaction" [19].

The $a_{i j}$ coefficients in this model represent interactions between populations as in the usual Lotka-Volterra models, but here are modified by the CN rules. 
For example, $a_{11}$ represents the effect of $x_{1}$ on its own growth rate (such as density-dependent mortality rates), and captures both direct and indirect competition for resources (see [6] and Supplementary Material for details). Similarly, $a_{12}$ represents the effect of $x_{2}$ on $x_{1}$ 's growth rate, both by predation and by sequestering resources that are then unavailable to $x_{1}$. The parameters $a_{21}$ and $a_{22}$ represent the effect of $x_{1}$ and $x_{2}$ respectively on $x_{2}$ 's growth rate, with $a_{21}$ representing growth due to ingestion of resources obtained from $x_{1}$ and $a_{22}$ representing intra-population competition such as density-dependent mortality. The $r_{i}$ terms represent the interactions with nonliving resources: in the case of the autotroph $x_{1}$ population, $r_{1}$ represents the net difference between growth on inorganic nutrient and losses due to density independent mortality. As $x_{2}$ is effectively a predator and does not grow on inorganic resources, $r_{2}$ represents the losses due to density-independent mortality.

The term $x_{2} /\left(x_{2}+\epsilon_{2}\right)$, that multiplies the $x_{1}$ population net growth term $r_{1}$, captures the dependence of the population on the presence of the $x_{2}$ population for pollination. The CN framework stipulates that the $x_{1}$ population must be able to grow when its pseudo-resource (its actual resource $N$ with its obliging population $x_{2}$ ) is maximum, and that it cannot grow when its pseudo-resource is zero. When $\epsilon_{2}=0, x_{1}$ does not depend on $x_{2}$ for pollination. The plant population $x_{1}$ is obligated on $x_{2}$ for pollination when $\epsilon_{2}$ is positive, but may receive no mutualist benefit (no mutualist interaction), no net mutualist benefit (i.e. the cost of attracting a benefit exceeds the benefit received), or may receive a weak or strong mutualist benefit. These scenarios depend on the relative magnitude and/or sign of $a_{12}$. The treatment of obligation in this way suggests that instead of $r_{1}$ being a net growth term, it should be partitioned out into a growth term that is dependent on $x_{2}$ and a loss term that is not. Such treatment introduces an Allee effect [1] which will be treated in a separate paper.

Mutualist benefits are represented in simple heuristic models such as (1) by indirect transfers of mass from the resource pool to the benefiting population that are mediated by the benefactor population. If the pollinator population just transports gametes within a small area and solely facilitates reproduction, then we might consider that no, or no net, mutualist benefit accrues to the plant. This is defined by the parameter relationship $a_{12}>r_{1}$. However, if the pollinator improves the overall fitness of the plant population, for example by increasing its geographical distribution, then the plant may additionally experience an increase in fitness from a weak $\left(0<a_{12}<r_{1}\right)$ or strong $\left(a_{12}<0\right)$ mutualist benefit (see the Supplementary Material and/or [6] for details).

The pollinator population in this simple model functions solely as a predator that consumes nectar and/or pollen supplied by the plant, but in the course of its predation pollinates the plant allowing reproduction. In contrast to the plant, whether the pollinator provides an additional mutualist benefit to the plant or not does not change the pollinator population's parameter profile (see below).

\section{Model Properties}

The zero isoclines, critical points and vector fields shown in Figure 1 summarise the dynamical properties of the pollination system for scenarios where the plant is dependent on a small pollinating population with no mutualism interaction Figure 
1(a), is dependent on a moderate pollinating population Figure 1(b), through the inclusion of a weak mutualism benefit Figure 1(c) to a strong mutualism benefit Figure $1(\mathrm{~d})$. The $f_{1}$ zero isocline is the curved line (barely evident in Figure 1(a)) with the upper branch that has a negative slope and the $f_{2}$ zero isocline is the straight line that always has a positive slope. The equilibrium points are defined by the intersections of these isoclines, with the stable equilibrium points indicated by the black dots. A key attribute of these diagrams is that the $f_{1}$ zero isocline starts at the origin and is constrained to lie within the ecospace $E$ (the regions in Figure 1 that contain arrows and we do not consider regions where $x_{1}$ and/or $x_{2}$ are negative) when no mutualist benefit accrues to the plant ( $\mathrm{a}$ and $\mathrm{b}$ ). However, the addition of mutualist benefits allows the upper branch of the $f_{2}$ isocline to extend outside $E$ by exiting through the "lid" $x_{1}+x_{2}=1$ (c and d). The positive slope of the upper branch of the zero isocline in Figure 1(d) is characteristic of the plant receiving a strong mutualist benefit. Note that the addition of an Allee effect, which as noted above occurs if a density independent mortality term is included in the plant equation (1), shifts the zero isocline up the axis resulting in the neutrally stable origin becoming a stable equilibrium.

Note that the parameter values used to draw Figure 1 represent a system that utilises only a small fraction of the available resources, as evidenced by the distance of the stable equilibrium point from the diagonal boundary of $E\left(x_{1}+x_{2}=1\right)$ in panels $(\mathrm{a}-\mathrm{c})$. This is not a common scenario: many systems, such as oceanic plankton systems, coral reefs and rainforests, sequester a significant fraction of total resources into living biomass and would have a stable equilibrium point much closer to the $x_{1}+x_{2}=1$ boundary of the ecospace. We use this rubric to investigate the potential for adaptation in pollination systems.

\section{Eco-evolution in Four Pollination Scenarios}

We used a genetic algorithm $[13,20]$ that maximised the total equilibrium biomass of the system $\left(x_{1}^{*}+x_{2}^{*}\right)$ to examine the outcomes of eco-evolutionary processes in four scenarios of obligate pollination interactions based on mutualist benefits that the pollinator confers on the pollinated population in addition to facilitating reproduction:

- No mutualism interaction (NM): the pollinator functions as a predator, that is, it consumes nectar or other rewards produced by the plant to attract it, but the plant receives no benefit from this apart from transport of gametes between plants that allows them to reproduce;

- No net mutualism benefit $(\mathrm{NN})$ : the pollinator provides a benefit to the plant in terms of increased fitness, but this benefit is less than the costs to fitness incurred in attracting the pollinator's services;

- Weak mutualism benefit (WM): the pollinator provides a benefit to the plant in terms of increased fitness that exceeds the costs to fitness incurred in attracting the pollinator, but does not exceed the reduction in available resources due to their sequestering by the pollinator population. The direct interaction for the plant is positive but the overall interaction (direct plus indirect) is negative;

- Strong mutualism benefit (SM): the pollinator provides a benefit to the plant in terms of increased fitness that greatly exceeds the costs incurred in attracting 

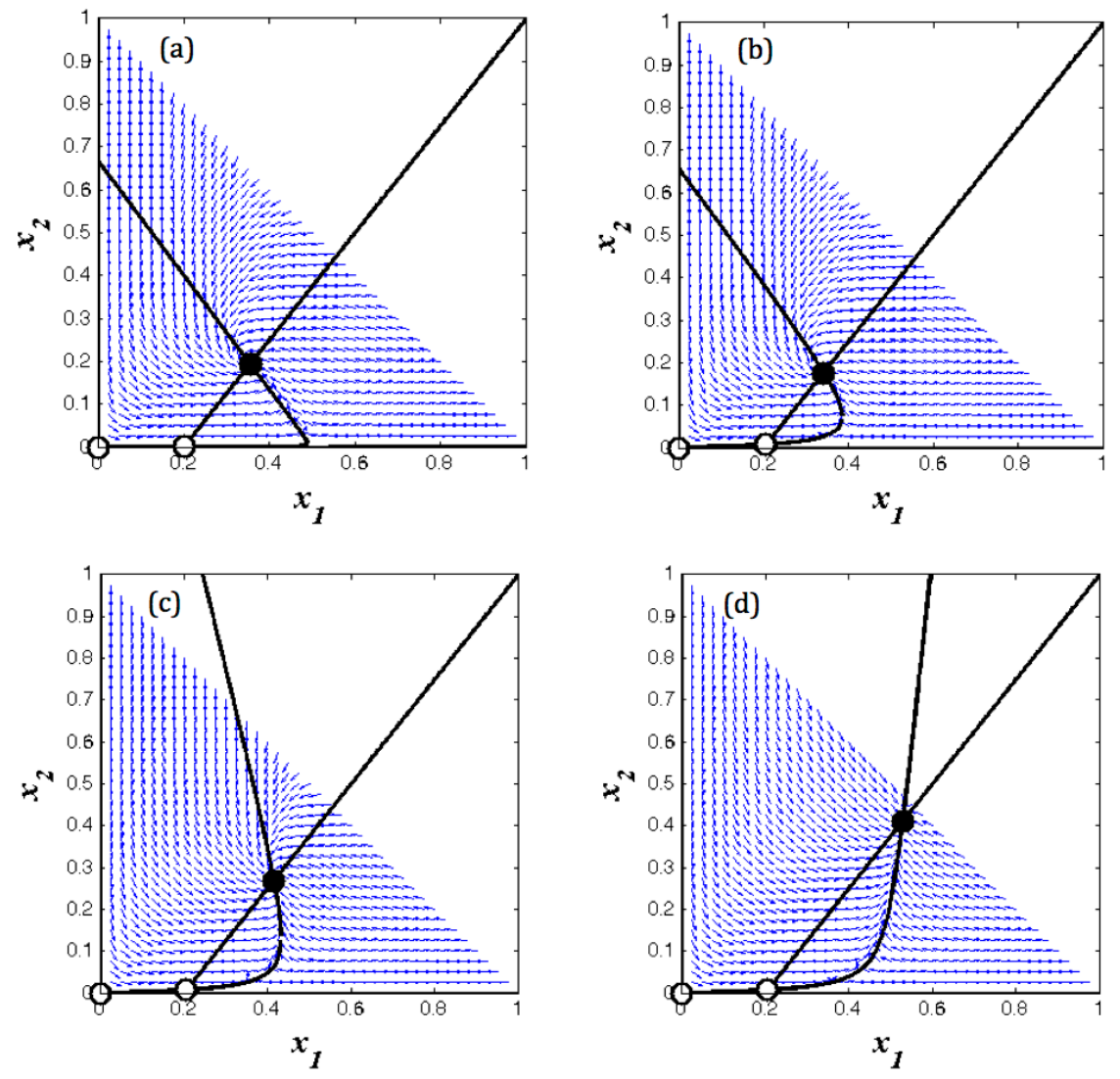

Fig. 1 Ecospace diagram with vector fields, zero isoclines, and equilibrium points for an obligate pollination mutualism. $x_{1}$ is a mutualist autotroph population that feeds on inorganic nutrient $N$ and is pollinated by the $x_{2}$ population that also predates on it. Stable equilibriums are shown by black dots, unstable ones by white dots. Panel: (a) obligate pollination dependent on a very small pollinating population $\left(\epsilon_{2}=0.0001 ; a_{12}=1.5\right)$; (b) pollination mutualism dependent on a small pollinator population $\epsilon_{2}=0.01 ; a_{12}=1.5$ ); (c) pollination mutualism with a weak mutualism benefit $\left(\epsilon_{2}=0.01 ; a_{12}=0.5\right) ;(\mathrm{d})$ pollination mutualism with a strong mutualism benefit $\left(\epsilon_{2}=0.01 ; a_{12}=-0.2\right)$. The other parameter values used to draw these figures are $r_{1}=1, r_{2}=-0.25, a_{11}=2, a_{21}=-1.25$, and $a_{22}=1$.

the pollinator, and exceeds the reduction in resource due to the presence of the pollinator. The overall interaction is positive for the plant.

The parameter relationships that define these interactions for the plant population $x_{1}$ are described by $a_{12}$ decreasing as we proceed through the cases:

$$
\begin{aligned}
& \text { NM: } \quad 0<r_{1}<a_{11}, a_{12}, \quad \text { with } \quad\left|a_{21}\right|<a_{12} \\
& \text { NN: } \quad 0<r_{1}<a_{11}, a_{12}, \\
& \text { WM: } \quad 0<a_{12}<r_{1}<a_{11}, \\
& \text { SM: } \quad a_{12}<0<r_{1}<a_{11} .
\end{aligned}
$$


The NM parameter relationships are defined by the usual resource-based constraints on an autotroph population's growth $\left(\left.f_{1}\right|_{\{0,0\}}>0,\left.f_{1}\right|_{\{1,0\}}<0\right.$, and $\left.\left.f_{1}\right|_{\{0,1\}}<0\right)$ and a heterotroph population's growth $\left(\left.f_{2}\right|_{\{0,0\}}<0,\left.f_{2}\right|_{\{1,0\}}>0\right.$, and $\left.\left.f_{2}\right|_{\{0,1\}}<0\right)$, with local mass balance $\left(\left|a_{21}\right|<a_{12}\right)$ as a predator cannot gain more biomass than its prey loses, see [6]. Local mass balance is relaxed for the NN case, to allow mutualism where costs may exceed benefits (see [14]). $x_{1}$ receives a net mutualism benefit from $x_{2}$, in addition to its obligation if $a_{12}<r_{1}$; we define weak mutualism to be the case where $r_{1}>a_{12}>0$, and strong mutualism to be the case where $a_{12}<0$.

We impose these discrete states NM - SM in our classification scheme only in order to examine the properties of each of these systems. In reality, the parameter boundaries that differentiate the states would be porous in the sense that systems might evolve through them. The states NM - SM are chosen to typify the existence and magnitude of mutualist interactions (in addition to obligate pollination), the states provide useful heuristic ecological value when considering the attributes of mutualism in these systems.

The parameter relationships for the pollinator population are the same for all scenarios and comply with the standard predator profile, which is $a_{21}<r_{2}<0<$ $a_{22}$, see [6] for details. The local mass balance condition that predators cannot gain more mass than their prey loses, forces $\left(a_{12}>\left|a_{21}\right|\right)$ in the NM case. The cases with mutualist benefits (WM and SM) require that the further constraint on parameters, that $\dot{x}_{1}+\dot{x}_{2}<0$ when $x_{1}+x_{2}=1[6]$ must be checked to ensure that sufficient resources to provide the mutualist benefits are actually available. This check is implemented during the evolution of the systems, and is used to discard ecologically unrealistic results.

We used the genetic algorithm to identify optimal parameter values when maximising "fitness" in several scenarios of obligate pollination interactions. Each run of the genetic algorithm evolved a population of 100 individuals (each individual represents a phenotype, a set of the six parameters $a_{11}, a_{12}, r_{2}, a_{21}, a_{22}$, and $\epsilon_{2}$ ) for 100 generations using a six-bit binary value to represent each parameter range (i.e. each individual in the genetic algorithm has a 36 bit genome). At each generation, each individual experienced a mutation probability for each bit and a cross-over probability for the genome that changed from an initial to a final probability according to a power law:

$$
P(m, c)_{n}=P(m, c)_{I}\left(\frac{P(m, c)_{F}}{P(m, c)_{I}}\right)^{\left(\frac{n}{N}\right)},
$$

where $P(m, c)_{n}$ is the probability for generation $n, P(m, c)_{I}$ is the initial probability, $P(m, c)_{F}$ is the final probability, and $N$ is the number of generations. The probability of a mutation $m$ of any gene at a generation reduced during the simulation from $P(m)_{I}=0.001$ to $P(m)_{F}=0.005$, while the probability of a cross-over increased from $P(c)_{I}=0.75$ to $P(c)_{F}=0.975$. Only one cross-over per genome per generation was allowed. The probability of each individual in each generation reproducing was defined using sigma-scaled roulette wheel selection [20] where the probability of an individual reproducing was correlated with its scaled fitness, defined by:

$$
F(r)_{i}=2+\frac{F_{i}-\bar{F}}{2 \sigma_{F}}
$$


Table 1 Parameter spaces searched for each scenario in the GA simulations. Note only $a_{12}$ changes between scenarios.

\begin{tabular}{ccccccccc}
\hline \multicolumn{7}{c}{ Scenario } \\
\hline Parameter & \multicolumn{7}{c}{ NM } & \multicolumn{7}{c}{ NN } & & WM & & SM \\
\hline & Min & Max & Min & Max & Min & Max & Min & Max \\
\hline$r_{1}$ & 1 & 1 & 1 & 1 & 1 & 1 & 1 & 1 \\
$a_{11}$ & 1.001 & 5.000 & 1.001 & 5.000 & 1.001 & 5.000 & 1.001 & 5.000 \\
$a_{12}$ & 1.001 & 2.000 & 1.001 & 2.000 & 0.000 & 1.000 & -3.000 & -0.001 \\
$r_{2}$ & -3.000 & -0.001 & -3.000 & -0.001 & -3.000 & -0.001 & -3.000 & -0.001 \\
$a_{21}$ & -4.000 & -0.001 & -4.000 & -0.001 & -4.000 & -0.001 & -4.000 & -0.001 \\
$a_{22}$ & 0.000 & 5.000 & 0.000 & 5.000 & 0.000 & 5.000 & 0.000 & 5.000 \\
$\epsilon_{2}$ & 0.001 & 0.100 & 0.001 & 0.100 & 0.001 & 0.100 & 0.001 & 0.100 \\
\hline
\end{tabular}

where $F(r)_{i}$ is the scaled fitness of individual $i, F_{i}$ is the measured fitness of $i$, assessed as the sum of the equilibrium biomasses $x_{1}^{*}$ and $x_{2}^{*}$ for the parameter set defined by individual $i, \bar{F}$ is the average measured fitness of the generation, and $\sigma_{F}$ is the variance of the measured fitness of the generation. Negative scaled fitnesses predicted by (4) were replaced by $F(r)_{i}=0.1$. The individual with the highest fitness always survived into the next generation.

Genetic algorithms require the specification of a goal function to estimate fitness during searches of the parameter space. We assume that each population ensures its survival by maximising its steady state population $[15,11,10]$, which suggests a goal function composed of the sum of the equilibrium populations. These are defined by the intersections of the $f_{1}$ and $f_{2}$ zero isoclines and are located at:

$$
\begin{aligned}
& x_{1}^{*}=\frac{r_{2}}{a_{21}}-\frac{a_{22}}{a_{21}} x_{2}^{*}, \quad \text { where } \\
& x_{2}^{*}=\frac{\beta \pm \sqrt{\beta^{2}+4 \alpha \gamma}}{2 \alpha} .
\end{aligned}
$$

where $\alpha=a_{11} a_{22}-a_{12} a_{21}, \beta=a_{11} r_{2}-a_{21} r_{1}-\epsilon_{2} \alpha$, and $\gamma=\epsilon_{2} a_{11} r_{2}$. Note that when $x_{1}^{*}=0$ the parameter definitions above result in $x_{2}^{*}=r_{2} / a_{22}$ lying outside the ecospace - this invalid solution is discarded. When $x_{2}^{*}=0,0<r_{2} / a_{21}=x_{1}^{*}=$ $r_{1} / a_{11}<1$.

The genetic algorithm searched the parameter spaces defined in Table 1, using 10,000 simulations of 100 generations of a population of 100 individuals for each scenario. In total, 400 million parameter sets were generated of which $262,685,511$ were valid, in that they complied with the criteria (2), plus other constraints $\left(0<x_{1}^{*}, x_{2}^{*}<1,0<x_{1}^{*}+x_{2}^{*}<1\right)$ that were implemented in the genetic algorithm.

The choice of goal function for the genetic algorithm to optimise the total biomass in each system simulates eco-evolutionary processes between the populations - as the parameter values that represent attributes of one population change, this may place selection pressure upon the other population to modify its parameters to increase total biomass. We note that while the genetic algorithm goal function has a maximum value everywhere on the line $x_{1}+x_{2}=1$, so we might expect the genetic algorithm to find a broad range of optima, with corresponding broad distributions of parameter values. However, this turns out not to be the case. 

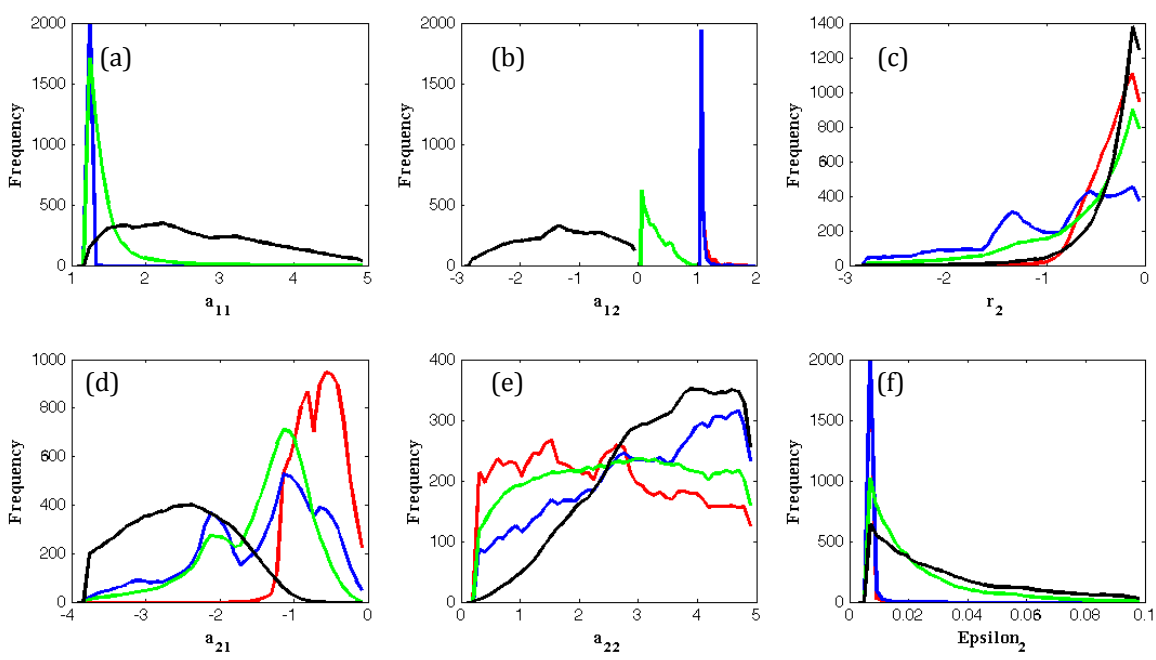

Fig. 2 Distributions of optimal parameter values found by the 10,000 simulations of 100 generations of 100 individuals for each of the four scenarios. The distributions are: NM (red, $70,540,356$ valid sets), NN (blue, 69,228,217 valid sets), WM (green, 62,980,994 valid sets) and SM (black, 59,935,944 valid sets). Some of the red distributions are not evident as they are obscured by the blue distributions. The distributions have been smoothed with a five-point moving average. Note that in panels (a), (b) and (f) the NM and NN distributions are very narrow spikes located at their leftmost bounds; the WM and SM distributions are not so constrained. The NM, WM and SM distributions are clearly constrained on the right in (c), as are the NM and SM distributions in (d) and (e) respectively. The others approximate uniform distributions in these panels, apart from SM which is mildly right-skewed in (d).

\section{Results}

The simulations for each scenario returned optimal parameter sets that resulted in close to the maximum possible value of the goal function $x_{1}^{*}+x_{2}^{*}=1$ as expected, with the parameter constraints imposed by the different trophic interaction scenarios resulting in different optimisations. The distributions of the 10,000 optimal parameter values found for each scenario are shown in Figure 2. This reveals that some scenarios have some parameters with very tight distributions, while some parameters have broad distributions in all scenarios. These variations in distributions suggest that some trade-offs between population attributes are inherent in optimising the total biomass in the system.

It is important to note that the minimum bounds of these parameters are the result of fundamental properties of the systems. The plant is obligate on the pollinator only if $\epsilon_{2}>0$, the plant has ecologically sensible attributes only if $a_{11}>r_{1}>0$, and receives no mutualist benefit from the pollinator only if $a_{12}>r_{1}$.

The distributions of optimal parameter values for $a_{11}, r_{2}, a_{21}, a_{22}$, and $\epsilon_{2}$ found by the genetic algorithm in Figure 2 have not been affected by arbitrary choices of parameter bounds - where distribution modes lie near the boundaries of the parameter spaces searched, these boundaries are fundamental attributes of the system. However, in order to investigate the properties of the various scenarios we have placed artificial boundaries on $a_{12}$.It is evident from Figure 2(b) that the 
selection pressures operating in the genetic algorithm would normally push the system through these boundaries in order to reach the overall optimal solution with $a_{12}<0$. The value of the artificial boundaries lies only in identifying heuristics associated with different systems that we classify.

\subsection{No Mutualist Benefit (NM and NN scenarios)}

The red and blue distributions in panels (a), (b), and (f) of Figure 2 reveal that the NM and NN scenarios are optimised with the minimum values for $a_{11}, a_{12}$, and $\epsilon_{2}$. This results in $a_{11} \approx a_{12} \approx 1=r_{1}$ and $\epsilon_{2}=0$ which leads to:

$$
f_{1} \approx 1-x_{1}-x_{2}=N
$$

The effect of this is to force the zero isocline near to the lid of the ecospace (Figure 3(a, b)), indicating that the total equilibrium biomass may be maximised (and conversely, the equilibrium nutrient minimised) at almost any point along the lid except near $x_{1}=1$. An optimum for this function may exist if there is curvature in the upper branch of the $f_{1}$ zero isocline that results in a close approach to the lid (see Supplementary Material). However, the genetic algorithm produces results that suggest that an optimum exists on the boundary of the ecospace, while the function is optimised at the vertex of the ecospace where $x_{2}=1$. However, $x_{2}=1$ is not a valid equilibrium solution for the system as it collapses to the origin, and Figure 3(c and d) reveal that in fact optima at the other extreme are more commonly found.

The parameter values that define the $f_{1}$ zero isoclines in the NM and NN scenarios describe plants that only interact with themselves and other populations through indirect competition for resources - the coefficients for the direct component of their interactions are close to zero (see [6] for details). This suggests that the costs to the plant of providing a reward/attractant such as nectar to its pollinator are close to zero, which appears implausible and is inconsistent with our fundamental assumption for the NN scenario that the costs of providing such a mutualist benefit exceed the benefits gained by the plant. These parameter values also dictate that the pollinator population must be small, as the plant provides very little resource to sustain the $x_{2}$ population - consequently $\epsilon_{2}$ must approximate zero to reflect that a very small pollinator population can service a very large plant population.

The goal function of the genetic algorithm may be maximised almost anywhere along the line $x_{1}+x_{2}=1$, which the $f_{1}$ zero isoclines closely approximate in the $\mathrm{NM}$ and NN scenarios, so we might expect the parameters that define the location and orientation of the $f_{2}$ zero isocline $\left(r_{2}, a_{21}\right.$, and $\left.a_{22}\right)$ in these scenarios to have broad distributions, and this is evident in Figure 2(c, d and e). We might expect substantial variation in the location of the intersection of the $f_{2}$ zero isocline with the $f_{1}$ zero isocline that determines the equilibrium population values. However, while Figure 3(b and $d$ ) confirm that the distributions of the optimal $f_{2}$ zero isoclines are much broader than those of the $f_{1}$ zero isoclines, the optimal $f_{2}$ zero isoclines generally intersect the $x_{1}$ axis (determined by $r_{2} / a_{21}$ ) near the origin and have very low slopes (determined by $a_{21} / a_{22}$ ). These characteristics lead to the $f_{1}$ and $f_{2}$ zero isoclines commonly intersecting in the vicinity of $\left\{x_{1}^{*} \approx 0.9, x_{2}^{*} \approx 0.1\right\}$ 

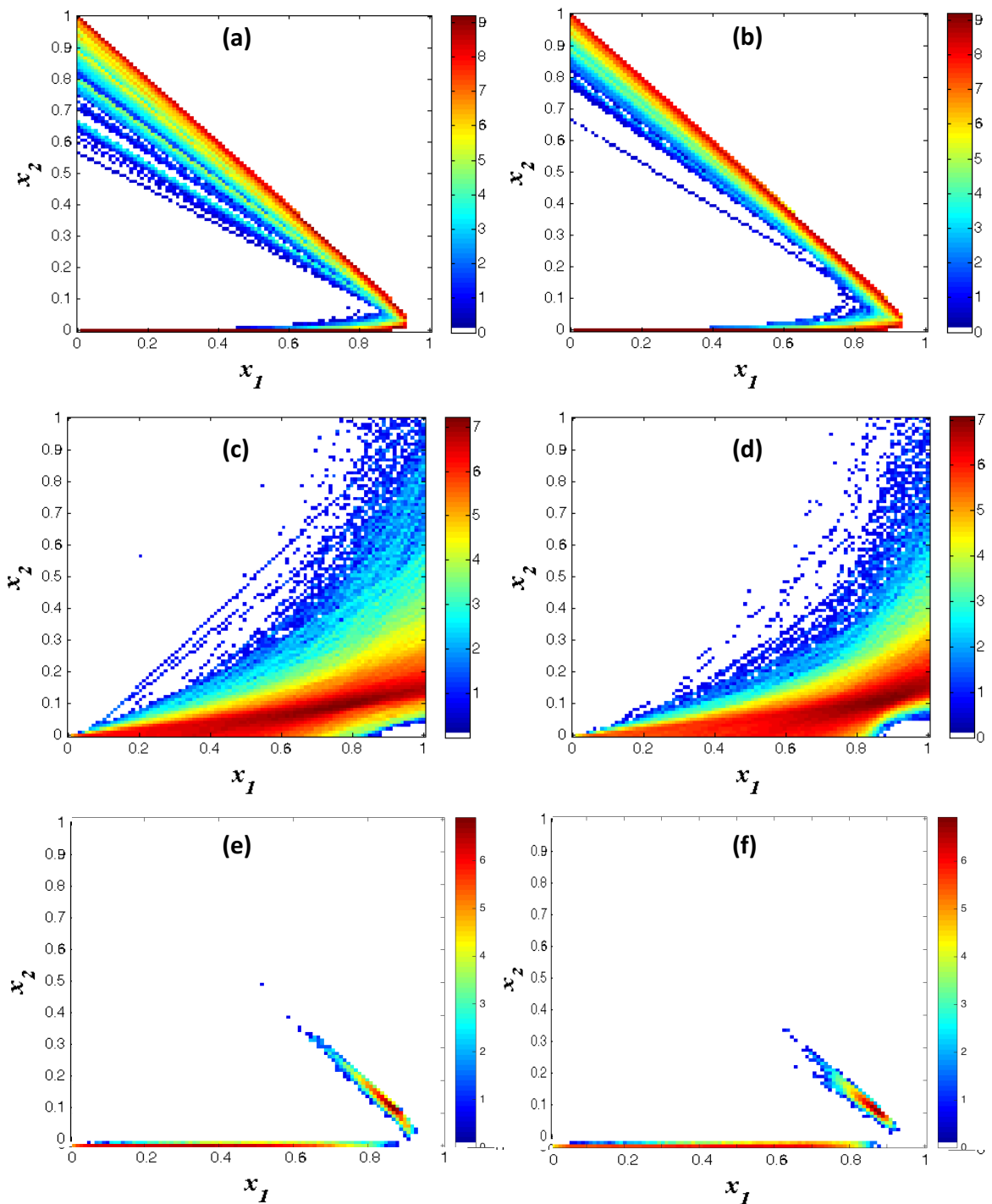

Fig. 3 Distributions of the $f_{1}$ (a and b) and $f_{2}$ (c and d) zero isoclines, and the equilibrium points (e and $\mathrm{f}$, where the $f_{1}$ and $f_{2}$ zero isoclines intersect) for the optimal parameter sets found for each of the 10,000 simulations for the NM (a, c and e) and NN (b, d and f) scenarios. The natural logarithms of the frequencies of occurrence of each isocline or equilibrium point are shown for each point in the ecospace. The equilibrium points near the $x_{1}$ axis are unstable, and the equilibrium points near the line $x_{1}+x_{2}=1$ are stable (see Figure 1). Note that each isocline is the result of the genetic algorithm selecting for maximum total biomass over 100 generations of a population of 100 individuals. 
(Figure 3(e and $\mathrm{f})$ ), consistent with a small value of $\epsilon_{2}$ indicating that the plant requires only a small pollinator population relative biomass in order to achieve high pollination rates.

The values of $r_{1}$ and $a_{21}$ were highly correlated (correlation coefficient $=0.98$ ) in both the NM and NN scenarios, with the relationships:

$$
\begin{aligned}
& r_{2} \approx 0.85 a_{21}+0.05, \quad(\mathrm{NM}), \\
& r_{2} \approx 0.87 a_{21}+0.11, \quad(\mathrm{NN}) .
\end{aligned}
$$

where we recall the constraint for these scenarios that $a_{21}<r_{1}<0$. These relationships constrain the maximum $x_{1}$ axis intercept of the $f_{2}$ zero isocline to be at $x_{1} \approx 0.85$ although Figure 3 (b and $\mathrm{d}$ ) reveal that the intercept most often occurs near $x_{1} \approx 0.2$. The distributions of the density-dependent mortality rates for the pollinator $\left(a_{22}\right.$, Figure $\left.2(\mathrm{e})\right)$ are relatively broad, reflecting that many values of $r_{2}$ and $a_{21}$ can provide an appropriate ratio to define intercepts near $x_{1} \approx 0.2$, allowing a number of $a_{22}$ values to optimise the total biomass in the system. Here, $a_{22}$ could also be interpreted as the ecology subsystem closure coefficient, representing the influence of predation by higher trophic levels.

\subsection{Weak and Strong Mutualist Benefit (WM and SM scenarios)}

The WM and SM scenarios (green and black lines in Figure 2), that include some net mutualism benefits, generally had broad optimal parameter distributions, with most parameters optimised at values in the interior of their parameter spaces. If the modal parameter values occur near the bounds of their domains (for example $r_{2}$ and $\epsilon_{2}$ ) the distributions are quite broad, reflecting weak selection pressures operating in the genetic algorithm. A key effect of allowing a net mutualist benefit for $x_{1}$ in addition to its obligation on $x_{2}$ for pollination, shown in Figure 2(c and d) and evident in Figure 4 ( $\mathrm{a}$ and $\mathrm{b}$ ), is that it relaxes the selection pressures on $a_{11}, a_{12}$, and $\epsilon_{2}$. The lower branch of this isocline always passes through the origin, and remains close to the $x_{1}$ axis for most results. However, the upper branch of the isocline is much less tightly constrained than in the NM and NN scenarios, with broad distributions of this branch evident in both the WM and SM scenarios. Recall that the key difference between the WM and SM scenarios is that the slope of the upper branch of the $f_{1}$ zero isocline is constrained to be negative in the WM scenario, and is constrained to be positive in the SM scenario.

The selection pressures operating on the $f_{2}$ zero isoclines in the genetic algorithm also appear weak in the WM and SM scenarios, with the distributions of optimal zero isoclines again much broader than in the NM and NN scenarios. However, Figure 4(c and d) reveal that these isoclines commonly intercept the $x_{1}$ axis closer to the origin and have increased slopes than in the NM and NN scenarios. This results in optima in which $x_{2}$ sequesters an increased share of the total resources and $x_{1}$ has a reduced proportion. The most common $f_{2}$ zero isoclines in the WM scenario are apparent in Figure 4(c) and produce an optimal biomass distribution in the vicinity of $\left\{x_{1}^{*} \approx 0.8, x_{2}^{*} \approx 0.2\right\}$, whereas in the SM scenario the optimal $f_{2}$ zero isoclines have a broader distribution, but lead to the most dense cluster of optimal biomass distribution equilibria of the four scenarios, near $\left\{x_{1}^{*} \approx 0.6, x_{2}^{*} \approx 0.4\right\}$ (Figure $4(\mathrm{f})$ ). 

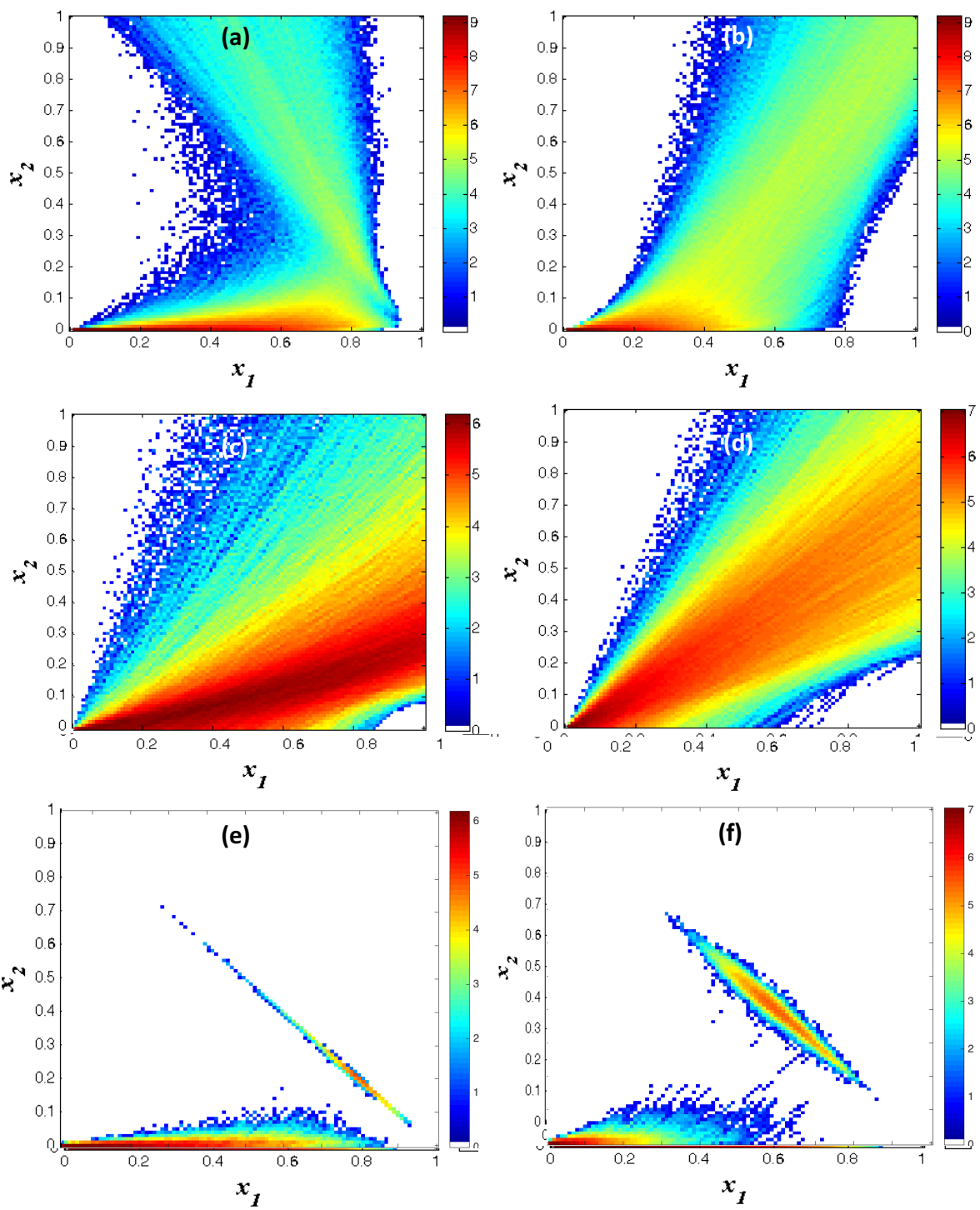

Fig. 4 Distributions of the $f_{1}$ (a and b) and $f_{2}$ (c and d) zero isoclines, and equilibrium points (e and $\mathrm{f}$, where the $f_{1}$ and $f_{2}$ zero isoclines intersect) for the optimal parameter sets found for each of the 10,000 simulations for the WM (a, c and e) and SM (b, d and f) scenarios. The equilibrium points near the $x_{1}$ axis are unstable, and the equilibrium points near the line $x_{1}+x_{2}=1$ are stable (see Figure 1). The natural logarithms of the frequencies of occurrence of each isocline are shown for each point in the ecospace. 
We note that, as in the NM and NN scenarios, the values of $r_{2}$ and $a_{21}$ are again highly correlated (correlation coefficient $=0.94$ ) in the WM scenario, with the relationship:

$$
r_{2} \approx 0.85 a_{21}+0.63
$$

where the constraint that $a_{21}<r_{2}<0$ still applies. However, they were not highly correlated (correlation coefficient $=0.25$ ) in the SM scenario. The highest correlation between parameter values in the SM scenario is between $a_{12}$ and $a_{21}$ (correlation coefficient $=0.92$ ), with the relationship:

$$
a_{12} \approx-2\left(a_{21}+1\right) .
$$

This reflects that the conservation of mass requirement constrains the amount of mutualist benefit that $x_{1}$ may accrue from $x_{2}$. This indicates a change in selection pressure in the SM scenario and reveals increased co-evolutionary selection pressures between $a_{12}$ and $a_{21}$ driving the genetic algorithm in this scenario.

\section{Discussion}

The simulation of selection pressures in the four obligate mutualism scenarios provides the interesting rubric that the greater the mutualism benefit the pollinator population provides to the plant in addition to its role in pollination, the more benefit, in terms of a larger share of resources, it accrues for itself. The simulations show that as the mutualism benefit received by the plant increases, its equilibrium population reduces and the equilibrium pollinator population size increases. This appears counter-intuitive as the net mutualism benefit, defined by $0<a_{12}<r_{1}$ (weak case) and $a_{12}<0$ (strong case) ultimately changes a loss term for the plant into a gain term. This represents the plant population acquiring an additional share of the total resources in the system reflecting an increase in the plant population's fitness due to it receiving a mutualist benefit from the pollinator. The facilitation of this benefit in scenarios WM and SM might be expected to increase the plant population's overall share of equilibrium resources, as it allows a term that was previously constrained to be a loss term in the NM and NN scenarios to become a gain term (all other parameters remain the same). However, the clear result of the isocline distributions in Figures 3 and 4 is that the result of the pollinator population providing a mutualist benefit to the plant population is instead to increase the pollinator equilibrium population. As the magnitude of the mutualism benefit is increased, from negative in the NM and NN scenarios through small positive values in the WM scenario to relatively large positive values in the SM scenario, the share of total resources sequestered by the pollinator equilibrium population $x_{2}^{*}$ progressively increases. Could this be an example of "enlightened generosity", where the pollinators are essentially "farming" the plants for their own gain?

A second interesting result is that the spread of both the optimal parameter and the zero isocline distributions increases as the mutualism benefit increases. While this allows for a greater variety of optimal solutions to the eco-evolutionary interactions, Figures 3 and 4 show that the selection pressures operating in the genetic algorithm favour certain solutions over others. Maximising the total biomass 
in the NM and NN cases involves minimising the plant population's dependence on the size of the pollinator population $\epsilon_{2}$ and its loss terms $a_{11}$ and $a_{12}$. Minimising these parameters describes a plant population that is effectively disengaged from direct interactions with other populations, only interacting through indirect competition for resources. This results in a plant zero isocline with an upper branch that approximates the boundary of the ecospace where little or no nutrient exists in nonliving form. This appears to allow the total biomass in the system to be maximised by a pollinating population with almost any set of attributes; however, this was not the case as some sets of attributes are observed more often than others. The primary eco-evolutionary process in these scenarios involves the pollinator's growth coefficient $a_{21}$ and its density-independent mortality $r_{2}$. The ratio of these parameters is instrumental in determining the plant equilibrium population $x_{1}^{*}$ (equation (3)), and dictates that the more the pollinator benefits from resources provided by the plant, the greater its density-independent mortality rate. This recycles nutrient back into the nutrient pool more rapidly, which is often correlated with increased resilience of ecosystems [9].

A similar trade-off between $a_{21}$ and $r_{2}$ is seen in the WM scenario, after the constraints on the $f_{1}$ zero isocline were lifted; however, when these constraints were relaxed further in the SM scenario, this trade-off was replaced by one between $a_{21}$ and $r_{2}$. This limited the amount of mutualism benefits available to $x_{1}$, a consequence of ensuring that local recycling and conservation of mass were not violated. The SM scenario led to the most dense concentration of stable equilibria and more stable equilibria that favoured large pollinator populations than any other scenario.

These results lead to a hypothesis that might be tested in real pollination ecosystems: that pollinators will in general provide net mutualism benefits to the plants they pollinate, in addition to the pure act of transporting gametes to facilitate reproduction, as it ultimately improves their own fitness. Pollination systems with these attributes will have a larger set of attributes available to them (analogous to broad distributions in our parameter space) that optimise the fitness of all populations in the system. Our results also suggest that plants will rely on relatively large populations of pollinators, with comparable shares of the active recycling resource, as this attribute improves the fitness of the whole system.

\section{Conclusions}

Competition, predation and mutualism are considered the tripod of population interactions upon which ecological analysis of communities rests $[5,16]$. Pollination is perhaps the most important of the mutualism interactions, as much of human agriculture depends on it; and gamete transport is one of the most common forms of mutualism between plants and insects/animals [30]. The coevolution of plants and animals involved in pollination interactions often focuses on morphological adaptations, with a recent study suggesting that finely-tuned eco-evolutionary states between a flower species and a pollinator species may be common [25]. Such results challenge the widespread view that pollinators are generalists, and suggest that obligate pollination interactions may be more common than previously thought. The development of a theoretical basis of obligate mutualism interactions has lagged those of other population interactions such as competition and predation, perhaps 
due to the apparent inability of simple Lotka-Volterra models, that have produced useful heuristics for competition and predation, to capture the essence of mutualism [22]. Our simulations suggest that plant/pollinator mutualist niches abound in the presence of obligated growth. When the pollinators provide further benefits to the plants, the pollinators can significantly improve their mutualist niche stability and their share of the available resources.

Lotka-Volterra models, that represent interactions between populations with simple linear functions that produce linear or planar zero isoclines or surfaces, arguably initiated the field of theoretical ecology in the 1920s, and have provided the basis for much of the basic theory we have today. We demonstrate here that even such simple models, when cast in a rigorous consumer-resource framework, can provide useful insights into subtle aspects of population interactions such as eco-evolution. Our results suggest the simple rubric that "it is better to give than to receive", or more specifically, that enlightened farming (as practiced by populations from ants to humans) is a successful evolutionary challenge to the view that nature is "red in tooth and claw" often used to characterise the drivers of evolution [7]. In contrast to the established view that competition is the dominant population interaction in the natural world, our rubric is consistent with the emerging view that most species on the planet are involved in mutualist interactions [5].

A caveat on our results is that we have only considered interactions between two populations. Analyses of mutualist networks [2,3] suggests that asymmetry may be a core attribute of these systems, that is, generalists mostly interact with specialists and vice versa. Mutualist networks often have a core of highly connected populations, upon which less-connected populations may depend. These attributes would suggest that while it may be beneficial for pollinators to provide plants with significant benefits, it may be better to provide many plants with benefits. The CN framework, that naturally places bounds on the quantum of such benefits and perforce imposes trade-offs by measuring all fluxes and resources in the same currency, provides a useful basis for such investigations. We expect the heuristics arising from $\mathrm{CN}$ systems to be broadly reflective of natural systems, as its primary approximation, that the key limiting resource that serves as the currency of the system is conserved and recycled, as most ecosystems recycle over $90 \%$ of their limiting resource [27].

\section{Acknowledgemnets}

The authors wish to thank Dr Andrew Morozov and the organisers of the organisers of the Modelling Biological Evolution 2017: Developing Novel Approaches International Conference held at the University of Leicester in April 2017.

\section{References}

1. Allee, W.C. AND E. S. Bowen, Studies in animal aggregations: mass protection against colloidal silver among goldfishes., The Journal of Experimental Biology, 61, 185-207, (1932).

2. Bascompte J., P. Jordano, C.J. Melia, and J.M. Olesen, The nested assembly of plant?animal mutualistic networks. PNAS 100(16): 9383-9387. (2003).

3. Bascompte J., P. Jordano, and J.M. Olesen, Asymmetric Coevolutionary Networks Facilitate Biodiversity Maintenance, Science 312: 431-433, (2006). 
4. Boucher, D.H., The Biology of Mutualism, Croom Helm, London, (1985).

5. Bronstein, J.L., Mutualism, Oxford University Press, New York, (2015).

6. Cropp, R.A. AND J. Norbury, Population interactions in ecology: a rule-based approach to modelling ecosystems in a mass-conserving framework, SIAM Review, 57, 437-465, (2015).

7. Dawkins, R., The Selfish Gene. Oxford University Press, Oxford. (1976).

8. DeAn, A.M., A simple model of mutualism., The American Naturalist, 121, 409-417, 1983.

9. DeAngelis D.L., Energy flow, nutrient cycling and ecosystem resilience. Ecology 61:764771 (1980).

10. de Mazancourt, C., M. Loreau and U. Dieckmann, Can the Evolution of Plant Defense Lead to Plant-Herbivore Mutualism? Am Nat 158(2): 109-123. 2001, p 115.

11. Dubois, D.M., Emergence of chaos in evolving Volterra ecosystems. in G. Van de Vijver, S.N. Salthe and M. Delpos (eds) Evolutionary Systems: Biological and Epistomological Perspectives on Selection and Self-Organisation. Springer-Science+Business Media. Dordrecht. pp197-215, 1998, p210.

12. W. G. Graves, B. Peckham And J. Pastor, A bifurcation analysis of a differential equations model for mutualism, Bulletin of Mathematical Biology, 68, 1851-1872, 2006.

13. J.H. Holland, Adaptation in natural and artificial systems, University of Michigan Press, Ann Arbour, 1975.

14. J. N. Holland And D. L. DeAngelis, A consumer-resource approach to the densitydependent population dynamics of mutualism, Ecology, 91, 1286-1295, 2010.

15. Jorgensen, S.E. And H.F. Mejer, Trends in Ecological Modelling. in Lauenroth, W.K. Skogerboe, G.V. and M. Flug (eds) Analysis of Ecological Systems: State-of-the-Art in Ecological Modelling. Elsevier, Amsterdam, pp 21-26. 1983, p 24.

16. S. Levin, The Princeton Guide to Ecology, Princeton University Press, Oxford, UK. 2012.

17. LotkA, A.J. Elements of Physical Biology Wilkins and Kilkins, Baltimore, 1925.

18. MACARTHUR RH Species packing and competitive equilibria for many species. Theoretical Population Biology 1:1-11(1970).

19. R.M. MAY, Theoretical Ecology: Principles and Applications, Blackwell Science Inc, Princeton, Oxford, UK, 1981.

20. M. Mitchell, An Introduction to Genetic Algorithms, MIT Press, Cambridge, Massachusetts, 1997.

21. M. Molles, Ecology: Concepts and Applications, McGraw-Hill Education,New York, 2014.

22. MurRAY JD Mathematical Biology vol 17. Interdisciplinary Applied Mathematics. Springer-Verlag, Berlin. (2001).

23. R.E. Ricklefs AND R. RelyeA, Ecology: the economy of nature, W.H. Freeman, New York, 2014.

24. L. L. Rockwood, Introduction to Population Ecology, Wiley-Blackwell, Oxford, 2015.

25. A. Shimizu, I. Dohzono, M. Nakaji, D. A. Roff, D. Miller, S. Osata, T. Yajima, S. Nittsu, N. Utsugi, T. Sugawara and J. Yoshinura, Fine-tuned Bee-Flower Coevolutionary State Hidden within Multiple Pollination Interactions, Scientific Reports, 4, doi:10.1038/srep03988, 2013.

26. Van der Meer J.H. And D.E. Goldberg, Population Ecology: First Principles, Princeton University Press, Woodstock, Oxfordshire, UK, 2013.

27. Vitousek P.M. and P.A. Matson, Nutrient cycling and biogeochemistry. In Levin S, editor. The Princeton Guide to Ecology. New Jersey: Princeton University Press, 2012. p 330-339.

28. VOLTERRA V Variations and fluctuations of the numbers of individuals in animal species living together. In: Reprinted in 1931 in Chapman RN (ed) Animal Ecology. McGraw Hill, New York, (1926)

29. Watson RA, Szathmary E How can evolution learn? Trends Ecology and Evoutionl 131:147-157. (2016).

30. Wilmer P Pollination and Floral Ecology. Princeton University Press, Princeton. (2011)

31. Zahran HH Rhizobium-Legume Symbiosis and Nitrogen Fixation under Severe Conditions and in an Arid Climate. Microbiol Mol Biol Rev. 63(4): 968?989. (1999) 